\title{
Efficient and simple one-pot conversion of resin-bound $N$-Fmoc amino acids and dipeptides into $N$-Boc derivatives
}

\author{
Ricardo L. E. Furlán ${ }^{a}$ and Ernesto G. Mata ${ }^{b}$ \\ ${ }^{\mathrm{a}}$ Area Farmacognosia - ${ }^{\mathrm{b}}$ Instituto de Química Orgánica de Síntesis (CONICET - UNR), \\ Facultad de Ciencias Bioquímicas y Farmacéuticas, Universidad Nacional de Rosario, Suipacha \\ 531, 2000 - Rosario, Argentina \\ E-mail: rfurlan@fbioyf.unr.edu.ar; emata@fbioyf.unr.edu.ar

\begin{abstract}
Dedicated to Professor Edmundo A. Rúveda on his $70^{\text {th }}$ birthday and Professor Roberto A. Rossi on his $60^{\text {th }}$ birthday

(received 15 Feb 03; accepted 10 Apr 03; published on the web 25 Apr 03)
\end{abstract}

\begin{abstract}
Wang resin bound $N$-fluorenylmethoxycarbonyl (Fmoc) amino acids and dipeptides were efficiently converted into the corresponding $N$-tert-butoxycarbonyl (Boc) derivatives using two different "one-pot" procedures: potassium fluoride / di-tert-butyl carbonate and potassium fluoride / tert-butyl S-(4,6-dimethylpyrimidin-2-yl) thiolcarbonate.
\end{abstract}

Keywords: Solid-phase synthesis, Boc carbamates, Fmoc carbamates, Amino acids and derivatives, dipeptides

\section{Introduction}

Despite the proliferation of protecting groups, functional group incompatibility remains a key issue in synthetic organic chemistry. ${ }^{1}$ The problem is particularly delicate in the design and synthesis of polyfuctional molecules such as peptides, oligosaccharides, glycopeptides, glycolipids, nucleotides, and polyketides.

The emergence of solid-phase chemistry ${ }^{2}$ has added a new element of complexity due to the different requirements and properties of the two phases. ${ }^{3}$ The $N$-fluorenylmethoxycarbonyl (Fmoc) and $N$-tert-butoxycarbonyl (Boc) groups are two of the most common protecting groups in solid-phase chemistry, with a large number of building blocks available containing them. ${ }^{4}$ They display contrasting chemical stabilities: $N$-Fmoc is usually cleaved by organic bases whereas $N$-Boc is stable to bases but removed in acidic medium. The increasing degree of sophistication of the solid-phase chemistry raises the problem of incompatibility of $\mathrm{N}$-Fmoc protection to different reagents. ${ }^{5}$ Moreover, $N$-Boc protecting group has proven to be better than $N$-Fmoc after five or six steps in the solid-phase synthesis of the "difficult" peptides, since acid 
deprotection may destroy undesirable $\beta$-sheet structures. ${ }^{6}$ Therefore, the development of solidphase methodologies for one-pot conversion of $\mathrm{N}$-Fmoc carbamates into $\mathrm{N}$-Boc carbamates under mild conditions is highly desirable.

A number of papers have appeared describing solution-phase examples of one-pot interconversion of carbamates, such as transformation of $N$-benzyloxycarbonyl (Cbz) group into Boc group ${ }^{7,8}$ or Fmoc group, ${ }^{9}$ conversion of $N$-fluorenylmethoxycarbamates into Boc group ${ }^{10}$ or Cbz group,${ }^{11}$ conversion of Boc into $\mathrm{Cbz},{ }^{12,13}$ and transprotection of $N$-allyloxycarbonyl (Aloc) to Boc group. ${ }^{14}$ (Scheme 1). However, the interconversion of carbamates in solid-phase chemistry is still unusual. ${ }^{15}$

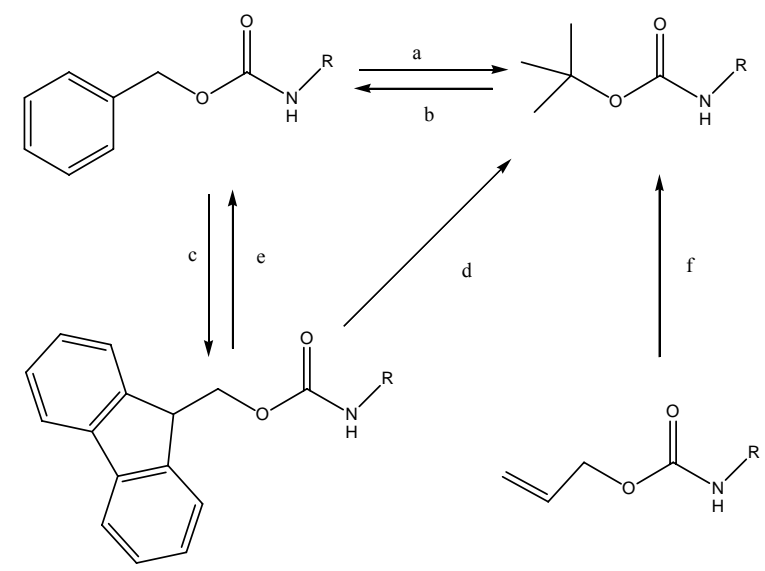

(a) Ref. 7 and 8, (b) Ref. 12 and 13, (c) Ref. 9, (d) Ref. 10, (e) Ref. 11, (f) Ref. 14.

Scheme 1. Solution-phase interconversion of carbamates.

In this publication, we describe the efficient one-pot conversion of Wang resin bound $\mathrm{N}$ Fmoc amino acids and dipeptides into the corresponding $N$-Boc derivatives. ${ }^{16}$

\section{Results and Discussion}

Transprotection reactions were carried out combining a base and a tert-butoxycarbonylating reagent. To cleave the Fmoc group we used fluoride ion; which has proven to be very efficient for the non-hydrolytic solution-phase cleavage of Fmoc carbamates. ${ }^{1,17}$ In addition, its presence in the reaction medium should not interfere with the tert-butoxycarbonylation step. We choose the potassium salt since it does not affect ester groups usually unstable in the presence of the generally used tetrabutylammonium fluoride. ${ }^{10}$

For reprotection we used three different ready-to-use tert-butoxycarbonylating agents: 1,2,2,2-tetrachloroethyl tert-butyl carbonate, ${ }^{18}$ di-tert-butyl dicarbonate, ${ }^{19}$ and tert-butyl S-(4,6dimethylpyrimidin-2-yl) thiolcarbonate (Boc-S). ${ }^{20}$ 
Monitoring reactions represents one of the major drawbacks of solid-phase chemistry. ${ }^{21}$ In order to examine the progress of the reaction, we first carried out the transprotection of FmocAla-OMe in solution. Using five equivalents of potassium fluoride and three equivalents of carbonylating agent (1,2,2,2-tetrachloroethyl tert-butyl carbonate or di-tert-butyl dicarbonate) in DMF yielded the Boc-protected product quantitatively in 5-7 hours at room temperature. Interestingly, no free amine was detected by TLC suggesting that deprotection of the amine group is the limiting step of the process rather than the Boc reprotection. Therefore, the amount of unreacted fluorenylmethoxycarbamate present in the reaction mixture can be regarded as an indication of the reaction progress.

Consequently, we next carried out the solid-phase transprotection of Fmoc-Phe-Wang resin using the combination potassium fluoride / di-tert-butyl dicarbonate in DMF. The amount of unreacted Fmoc group at different times was determined as "Fmoc loading" in small portions of resin by cleaving the carbamate with piperidine followed by UV quantitation of the resulting piperidine-dibenzylfulvene adduct at $301 \mathrm{~nm}{ }^{22}$ In this way, it was possible to estimate the reaction time required for complete interconversion of the Fmoc group into Boc group (Figure 1).
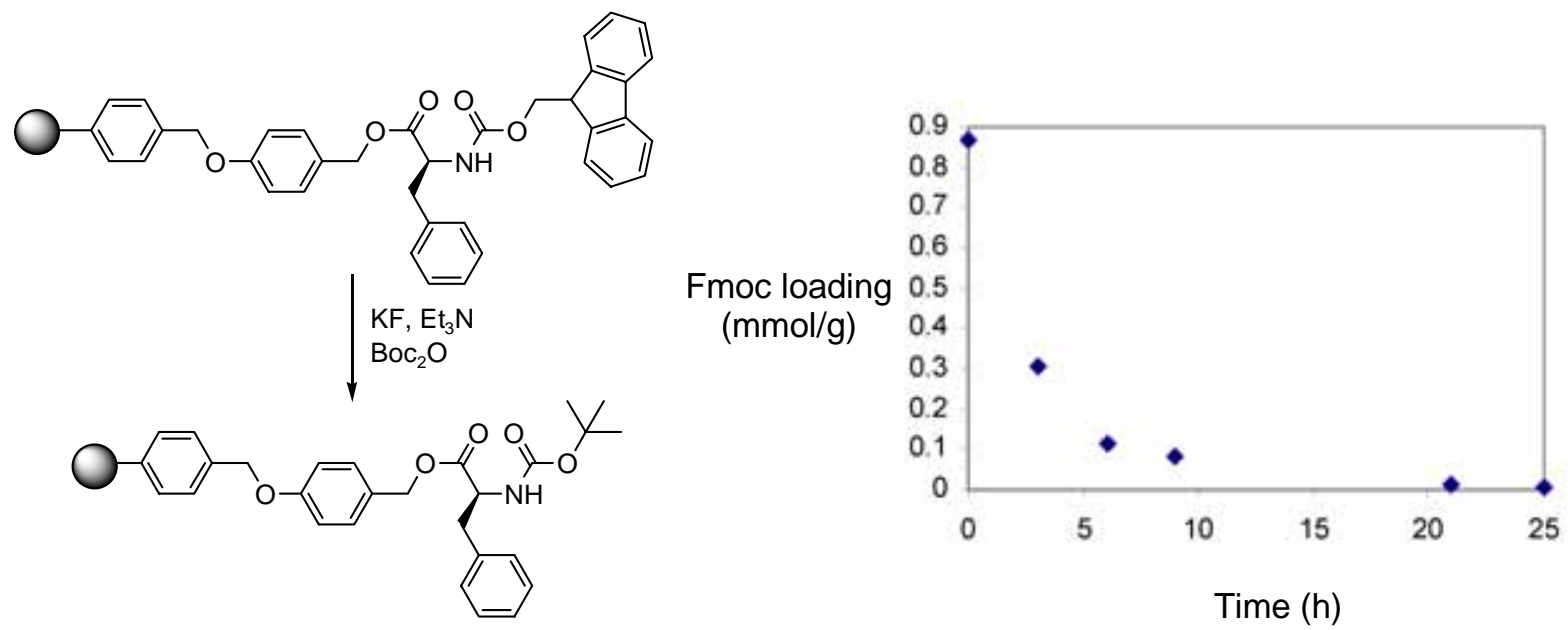

Figure 1. Solid-phase transprotection of Fmoc-Phe-Wang resin. UV determination of unreacted Fmoc group on the resin.

To corroborate that the deprotected amine had in effect been reprotected in situ as Boccarbamate, the product was detached from the solid support using trimethyltin hydroxide (TMTOH), which produces the selective cleavage of the benzyl ester link to the resin without affecting the tert-butoxycarbamate (Scheme 2). ${ }^{23,24}$ The yield for the entire process (Fmoc deprotection, Boc protection, and cleavage from the solid support) was $95 \%$.

In order to evaluate the scope of the reaction, the methodology was applied to the solid phase transprotection of a variety of Wang resin-bound amino acids and dipeptides (Table 1). Using the combination $\mathrm{KF} / \mathrm{Boc}_{2} \mathrm{O}$, the Fmoc carbamates were smoothly converted into Boc carbamates in high overall yields for the isolated products. 


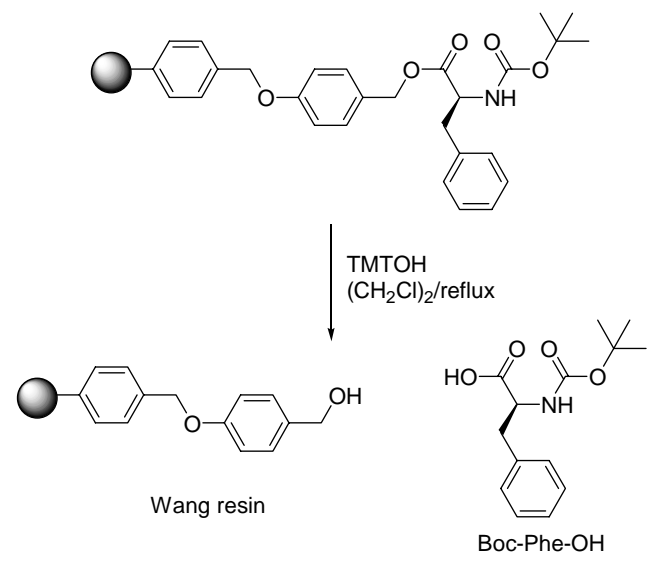

Scheme 2. Final detachment of Boc-Phenylalanine from Wang resin.

Table 1. Solid-phase conversion of Fmoc carbamates into Boc carbamates ${ }^{a}$

\begin{tabular}{|c|c|c|c|c|}
\hline Entry & Substrate & Product & $\begin{array}{c}\mathrm{KF} / \mathrm{Boc}_{2} \mathrm{O} \\
\text { Yield }(\%)^{\mathrm{b}}\end{array}$ & $\begin{array}{l}\text { KF/Boc-S } \\
\text { Yield }(\%)^{\mathrm{b}}\end{array}$ \\
\hline 1 & Fmoc-Ala-Wang resin & Boc-Ala-OH & 94 & 100 \\
\hline 2 & Fmoc-Leu-Wang resin & Boc-Leu-OH & 96 & 100 \\
\hline 3 & Fmoc-Ser $\left(\mathrm{O}^{t} \mathrm{Bu}\right)$-Wang resin & $\mathrm{Boc}-\mathrm{Ser}\left(\mathrm{O}^{t} \mathrm{Bu}\right)-\mathrm{OH}$ & 100 & 85 \\
\hline 4 & Fmoc-Boc-Lys-Wang resin & $(\mathrm{Boc})_{2}-\mathrm{Lys}-\mathrm{OH}$ & 86 & 80 \\
\hline 5 & Fmoc-Cys(p-MeOBn)-Wang resin & Boc-Cys ( $p-\mathrm{MeOBn})-\mathrm{OH}$ & 88 & 100 \\
\hline 6 & Fmoc-Pro-Wang resin & Boc-Pro-OH & 72 & 57 \\
\hline 7 & Fmoc-Phe-Wang resin & Boc-Phe-OH & 95 & 100 \\
\hline 8 & resin & & 100 & 100 \\
\hline 9 & & & 74 & 83 \\
\hline 10 & & & 81 & 76 \\
\hline 11 & & & 64 & 78 \\
\hline
\end{tabular}

${ }^{a}$ All reactions were carried out according to the general procedure described in experimental section. ${ }^{\mathrm{b}}$ Overall isolated yield, based on the initial loading level of Fmoc-AA-Wang resin or Fmoc-AA-AA-Wang resin (three steps). 
Functionalities such as tert-butyl ether, $p$-methoxybenzyl thioether, and amide were stable to the reaction conditions. No loss of enantiomeric purity was detected after comparing optical rotations of representative products with reported values.

Among the various reagents proposed to effect Boc protection, tert-Butyl S-(4,6dimethylpyrimidin-2-yl) thiolcarbonate (Boc-S) has been widely recognised as the better alternative to $\mathrm{Boc}_{2} \mathrm{O}$. In order to test its capability to perform the solid-phase reprotection of the amine group, the same set of Wang resin-bound amino acids and dipeptides were treated with the combination of KF / Boc-S (Table 1). Yields were similar to those previously achieved with $\mathrm{Boc}_{2} \mathrm{O}$, indicating that both tert-butoxycarbonylating agents can be used indiscriminately. Again, no sign of racemization was detected.

Despite the promising results in solution-phase studies, our attempts to use 1,2,2,2tetrachloroethyl tert-butyl carbonate for the solid-phase tert-butoxycarbonylation were unsuccessful. When the Fmoc-Ala-Wang resin was treated through a one-pot procedure with the tamdem KF / 1,2,2,2-tetrachloroethyl tert-butyl carbonate, no Fmoc carbamate was detected on the solid support after $24 \mathrm{~h}$. However, Boc reprotection did not occur and the final detachment of the resin-bound product with TMTOH mainly yielded $N$-deprotected alanine.

\section{Conclusions}

In summary, we describe an efficient methodology for the one-pot conversion of Wang resin bound $N$-Fmoc amino acids and dipeptides into the corresponding $N$-Boc derivatives, using two different tert-butoxycarbonylating agents: di-tert-butyl dicarbonate $\left(\mathrm{Boc}_{2} \mathrm{O}\right)$ and tert-butyl S(4,6-dimethylpyrimidin-2-yl) thiolcarbonate (Boc-S). This approach could find applicability not only for those wishing to synthesize peptide residues appropriate to solid-phase peptide synthesis (SPPS), but also in the field of combinatorial chemistry, particularly in the solid-phase synthesis of small molecule libraries. The Boc protecting group has some advantages compared to the Fmoc protecting group due to its stability under basic conditions. Replacement of an Fmoc carbamate by a Boc carbamate could be necessary and a one-pot conversion would facilitate this process by eliminating additional reaction steps.

\section{Experimental Section}

General Procedures. NMR spectra were measured in $\mathrm{CDCl}_{3}$ or $\mathrm{DMSO}_{-} \mathrm{d}_{6}$ at $200 \mathrm{MHz}$ for protons and $50 \mathrm{MHz}$ for ${ }^{13} \mathrm{C}$, and recorded on a Bruker AC-200 spectrometer. Infrared spectra were taken on a Beckman AccuLab 8 spectrophotometer. Optical rotations were measured with a Jasco DIP-1000 polarimeter at ambient temperature using a 1-mL capacity cell. UV absorbance was measured on a Beckman DU 640 spectrophotometer. TLC and spectral analyses (IR, ${ }^{1} \mathrm{H}$ and ${ }^{13} \mathrm{C}$ NMR) indicated that products were identical with commercial authentic material. $N$ - 
protected amino acids were purchased from Calbiochem-Novabiochem Corp. Fmoc-dipeptides were synthesized using conventional Fmoc-chemistry.

\section{Solid phase transprotection of Fmoc group into Boc group}

$\mathbf{K F} / \mathbf{B o c}_{2} \mathbf{O}$. Typical procedure. To a stirred suspension of Fmoc-AA-Wang resin (0.099 mmol) in DMF (1.3 mL) under nitrogen, KF (59 mg, $1.01 \mathrm{mmol})$ and $\mathrm{Et}_{3} \mathrm{~N}(31 \mu \mathrm{L}, 0.22 \mathrm{mmol})$ were added. The mixture was kept at room temperature and di-tert-butyl dicarbonate $\left(\mathrm{Boc}_{2} \mathrm{O}\right)(28.37$ $\mathrm{mg}, 0.13 \mathrm{mmol}$ ) was added. The reaction mixture was stirred until no Fmoc attached to the resin was detected by UV (24 h). The resin was filtered out, washed successively with DMF, $\mathrm{HCl} 1 \mathrm{~N}$, $5 \% \mathrm{NaHCO}_{3}, \mathrm{H}_{2} \mathrm{O}, \mathrm{MeOH}, \mathrm{AcOEt}, \mathrm{CHCl}_{3}$ and $\mathrm{MeOH}$, and dried overnight at reduced pressure.

KF/Boc-S. Typical procedure.To a stirred suspension of Fmoc-AA-Wang resin (0.078 mmol) in DMF (1.5 mL) under nitrogen, $\mathrm{KF}(39 \mathrm{mg}, 0.67 \mathrm{mmol})$ and $\mathrm{Et}_{3} \mathrm{~N}(40 \mu \mathrm{L}, 0.234 \mathrm{mmol})$ were added at room temperature. Then, a solution of tert-butyl S-(4,6-dimethylpyrimidin-2-yl) thiolcarbonate (Boc-S) $(58.1 \mathrm{mg}, 0.284 \mathrm{mmol})$ in DMF $(1 \mathrm{~mL})$ was added. The reaction mixture was stirred until no Fmoc attached to the resin was detected by UV ( $24 \mathrm{~h})$. The resin was filtered out, washed successively with DMF, $\mathrm{HCl} 1 \mathrm{~N}, 5 \% \mathrm{NaHCO}_{3}, \mathrm{H}_{2} \mathrm{O}, \mathrm{MeOH}, \mathrm{AcOEt}, \mathrm{CHCl}_{3}$ and $\mathrm{MeOH}$, and dried overnight at reduced pressure.

The transprotection reaction was monitored by estimating the amount of unreacted Fmoc on the resin expressed as Fmoc loading.

Typical procedure. 3-8 $\mathrm{mg}$ of resin were filtered out and washed with DMF, $\mathrm{HCl} \mathrm{1N,5 \%}$ $\mathrm{NaHCO}_{3}, \mathrm{H}_{2} \mathrm{O}, \mathrm{MeOH}, \mathrm{AcOEt}, \mathrm{CHCl}_{3}$ and $\mathrm{MeOH}$. The resin was dried at reduced pressure and accurately weighed out into a measuring flask. 20\% piperidine in DMF (25 mL) was added, the resulting mixture was stirred for $20 \mathrm{~min}$ and centrifuged. Three readings of the $300 \mathrm{~nm}$ absorbance of the supernatant were taken and their average was introduced in the following equation to obtain the Fmoc loading of the resin.

$$
\frac{\text { UV reading } \times 25 \times 1000}{7800 \times \text { wt of resin in } \mathrm{mg}}=\text { Fmoc loading in } \mathrm{mmol} / \mathrm{g} \text { of resin }
$$

\section{Cleavage of Boc protected amino acids and dipeptides}

Typical procedure. To a stirred suspension of resin $(0.22 \mathrm{mmol}$ of Fmoc protected amino acid or dipeptide) in $2.5 \mathrm{~mL}$ of $\left(\mathrm{CH}_{2} \mathrm{Cl}\right)_{2}$ was added TMTOH $(0.154 \mathrm{~g}, 0.56 \mathrm{mmol})$ at room temperature under nitrogen. The reaction mixture was then refluxed $\left(83^{\circ} \mathrm{C}\right)$ for $9 \mathrm{~h}$ and the resulting suspension filtered and washed succesively with $\left(\mathrm{CH}_{2} \mathrm{Cl}\right)_{2}, \mathrm{CH}_{2} \mathrm{Cl}_{2}, \mathrm{MeOH}$ and AcOEt. The combined organic solution was evaporated to dryness under reduced pressure and the resultant residue was dissolved in $20 \mathrm{~mL}$ of AcOEt and washed succesively with diluted aqueous solution of $\mathrm{HCl}(5 \times 6 \mathrm{~mL})$, water $(1 \times 6)$ and Brine $(1 \times 6)$. Therefore, it was extracted with 5\% $\mathrm{NaHCO}_{3}$ solution $(3 \times 7 \mathrm{~mL}$ ), the combined aqueous solution was acidified to $\mathrm{pH} 3-4$ with $2.5 \mathrm{~N}$ $\mathrm{H}_{2} \mathrm{SO}_{4}$ solution and extracted with $\operatorname{AcOEt}(3 \times 11 \mathrm{~mL})$. The AcOEt solution was dried $\left(\mathrm{Na}_{2} \mathrm{SO}_{4}\right)$ and evaporated to dryness. In all cases the ${ }^{1} \mathrm{H}$ NMR analysis of the crude reaction product 
showed only the corresponding $N$ - $\alpha$-Boc-L-amino acid or dipeptide. No other product was detected by ${ }^{1} \mathrm{H}$ NMR.

$N$ - $\alpha$-Boc-L-alanine. see ref. 24.

$N$ - $\alpha$-Boc-L-leucine. $[\alpha]^{20}$ D -29.8 (c 1.0 in AcOH) [lit. ${ }^{25}-30$ (c 1.0 in AcOH)]. IR (film) v 3340, $3320(\mathrm{~N}-\mathrm{H}$, urethane), $3000(\mathrm{O}-\mathrm{H}, \mathrm{COOH}), 1760(\mathrm{v}=\mathrm{O}$, carboxylic, dimer $), 1710(\mathrm{C}=\mathrm{O}$, carboxylic, monomer), $1680\left(\mathrm{C}=\mathrm{O}\right.$, urethane), $1530 \mathrm{~cm}^{-1}$ (amide II band). ${ }^{1} \mathrm{H} \mathrm{RMN}\left(\mathrm{CDCl}_{3}\right) \delta$ 0.96 (d, 6H, J = 6.2 Hz, Me), 1.45 (s, 9H, Me), 1.40-1.85 (m, 3H, $\left.\mathrm{CH}_{2}, \mathrm{CH}\right), 4.30$ (br s, 1H, CH), 4.90 (br s, $1 \mathrm{H}, \mathrm{NH}) .{ }^{13} \mathrm{C} \mathrm{RMN}\left(\mathrm{CDCl}_{3}\right) \delta 21.7,22.7(\mathrm{C} \delta), 24.66(\mathrm{C} \gamma), 28.18(\mathrm{Me}, \mathrm{Boc}), 41.37$ $(\mathrm{C} \beta), 51.98(\mathrm{C} \alpha), 80.05(\mathrm{C}, \mathrm{Boc}), 156.00(\mathrm{C}=\mathrm{O}, \mathrm{Boc}), 177.86(\mathrm{COOH})$.

$\mathrm{N}$ - $\alpha$-Boc-O-tert-butyl-L-serine. IR (film) v $3000(\mathrm{O}-\mathrm{H}, \mathrm{COOH}), 1730-1660(\mathrm{C}=\mathrm{O}, \mathrm{COOH}$ and urethane), $1530 \mathrm{~cm}^{-1}$ (amide II band). ${ }^{1} \mathrm{H} \mathrm{RMN}\left(\mathrm{CDCl}_{3}\right) \delta 1.20$ (s, 9H, Me), 1.46 (s, 9H, Me), $3.54\left(\mathrm{dd}, \mathrm{AB}\right.$ system, $J=8.80$ and $\left.5.4 \mathrm{~Hz}, 1 \mathrm{H}, \mathrm{CH}_{2}\right), 3.84(\mathrm{dd}, \mathrm{AB}$ system, $J=8.80$ and 5,3 Hz, $\left.1 \mathrm{H}, \mathrm{CH}_{2}\right), 4.37$ (br s, $\left.1 \mathrm{H}, \mathrm{CH}\right), 5.34(\mathrm{~d}, J=7.82 \mathrm{~Hz}, 1 \mathrm{H}, \mathrm{NH}) .{ }^{13} \mathrm{C} \mathrm{RMN}\left(\mathrm{CDCl}_{3}\right) \delta 27.14(\mathrm{Me}$, t-Bu), $28.17(\mathrm{Me}, \mathrm{Boc}), 53.82(\mathrm{C} \alpha), 61.62(\mathrm{C} \beta), 73.90(\mathrm{C}, t-\mathrm{Bu}), 80.05(\mathrm{C}, \mathrm{Boc}), 155.65(\mathrm{C}=\mathrm{O}$, Boc), $175.06(\mathrm{COOH})$.

$\mathbf{N}_{\alpha}$-Boc-N $\mathbf{N}_{\varepsilon}$-Boc-L-lysine. IR (film) v $3370(\mathrm{~N}-\mathrm{H}), 3000(\mathrm{O}-\mathrm{H}, \mathrm{COOH}), 1730(\mathrm{C}=\mathrm{O}, \mathrm{COOH})$, $1700\left(\mathrm{C}=\mathrm{O}\right.$, urethane), $1530 \mathrm{~cm}^{-1}$ (amide II band). ${ }^{1} \mathrm{H} \mathrm{RMN}\left(\mathrm{CDCl}_{3}\right) \delta 1.44(\mathrm{~s}, 18 \mathrm{H}, \mathrm{Me}), 1.40-$ 1.90 (m, 6H, $\mathrm{CH}_{2}$ ), 3.11 (br s, $2 \mathrm{H}, \mathrm{CH}_{2}$ ), 4.13 (br s, $1 \mathrm{H}, \mathrm{CH}$ ), 4.80 (br s, $1 \mathrm{H}, \mathrm{NH}$ ), 5.44 (br s, $1 \mathrm{H}$,

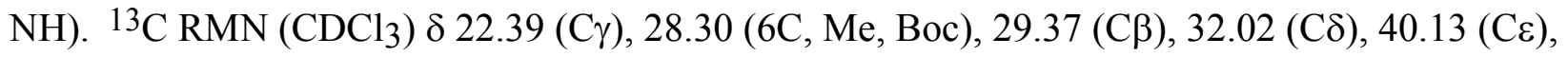
$53.43(\mathrm{C} \alpha), 79.18$ (C, Boc), $79.75(\mathrm{C}, \mathrm{Boc}), 155.66(\mathrm{C}=\mathrm{O}, \mathrm{Boc}), 156.00(\mathrm{C}=\mathrm{O}, \mathrm{Boc}), 175.97$ $(\mathrm{COOH})$.

$N$ - $\alpha$-Boc-S-p-methoxybenzyl-L-cysteine. see ref. 24.

$N$ - $\alpha$-Boc-L-proline. see ref. 24.

$N$ - $\alpha$-Boc-L-phenylalanine. $[\alpha]^{20}{ }_{\mathrm{D}}+23.7$ (c 1.5 in EtOH) $\left[\operatorname{lit}^{25}+23.7\right.$ (c 1.5 in EtOH)]. IR (film) v 3340-3300 (N-H, urethane), $3000(\mathrm{O}-\mathrm{H}, \mathrm{COOH}), 1735-1660 \mathrm{~cm}^{-1}(\mathrm{C}=\mathrm{O}, \mathrm{COOH}$, urethane). ${ }^{1} \mathrm{H} \mathrm{RMN}\left(\mathrm{CDCl}_{3}\right) \delta 1.40(\mathrm{~s}, 9 \mathrm{H}, \mathrm{Me}), 2.90-3.15\left(\mathrm{~m}, 2 \mathrm{H}, \mathrm{CH}_{2}\right), 4.60$ (br s, 1H, CH), 4.95 (br s, 1H, NH), 7.22 (m, 5H, Ar). ${ }^{13} \mathrm{C} \mathrm{RMN}\left(\mathrm{CDCl}_{3}\right) \delta 28.13$ (Me, Boc), 37.71and 38.98 $(\mathrm{C} \beta), 54.21$ and $55.95(\mathrm{C} \alpha), 80.11$ and 81.39 (C, Boc), $126.86(-\mathrm{CH}, \mathrm{Ar}), 128.41$ (2C, $\mathrm{CH}, \mathrm{Ar})$, 129.29 (2C, $\mathrm{CH}, \mathrm{Ar}), 135.82$ and $136.34(\mathrm{C}, \mathrm{Ar}), 155.27$ and $156.31(\mathrm{C}=\mathrm{O}, \mathrm{Boc}), 175.85$ and $176.19(\mathrm{COOH})$. (double signals are observed because of hydrogen bond formation).

$N$ - $\alpha$-Boc-L-proline-L-phenylalanine. IR (film) $v 3000(\mathrm{O}-\mathrm{H}, \mathrm{COOH}), 1720-1660 \mathrm{~cm}^{-1}(\mathrm{C}=\mathrm{O}$, $\mathrm{COOH}$ and urethane). ${ }^{1} \mathrm{H} \mathrm{RMN}\left(\mathrm{CDCl}_{3}\right) \delta 1.40(\mathrm{~s}, 9 \mathrm{H}, \mathrm{Me}), 1.70-2.10\left(\mathrm{~m}, 4 \mathrm{H}, \mathrm{CH}_{2}\right), 3.00-3.45$ (m, 4H, $\mathrm{CH}_{2}-\mathrm{N}, \mathrm{CH}_{2}-\mathrm{Ph}$ ), 4.28 (br s, $\left.1 \mathrm{H}, \mathrm{CH}\right), 4.86$ (m, 1H, CH), 7.23 (m, 6H, Ph, NH). ${ }^{13} \mathrm{C}$

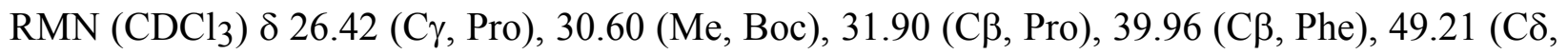

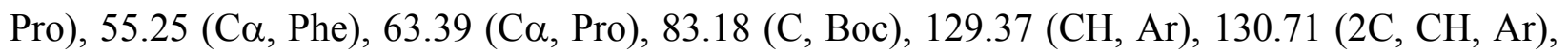
131.59 (2C, CH, Ar), 138.51 (C, Ar), 157.78 (C=O, Boc), 174.77 and $176.39(\mathrm{C}=\mathrm{O})$.

$\mathrm{N}$ - $\alpha$-Boc-O-tert-butyl-L-serine-L-phenylalanine. ${ }^{1} \mathrm{H}$ RMN $\left(\mathrm{CDCl}_{3}\right) \delta 1.14(\mathrm{~s}, 9 \mathrm{H}, \mathrm{Me}), 1.43$ (s, 9H, Me), 3.13 (m, 2H, $\mathrm{CH}_{2}$ ), 3.38 (br s, 1H, $\mathrm{CH}_{2}$ ), 3.74 (br s, $1 \mathrm{H}, \mathrm{CH}_{2}$ ), 4.16 (br s, $1 \mathrm{H}, \mathrm{CH}$ ), 4.86 (br s, $1 \mathrm{H}, \mathrm{CH}), 5.44$ (br s, $1 \mathrm{H}, \mathrm{NH}), 7.15-7.30$ (m, 5H, Ph). ${ }^{13} \mathrm{C} \mathrm{RMN}\left(\mathrm{CDCl}_{3}\right) \delta 27.19(\mathrm{Me}$,

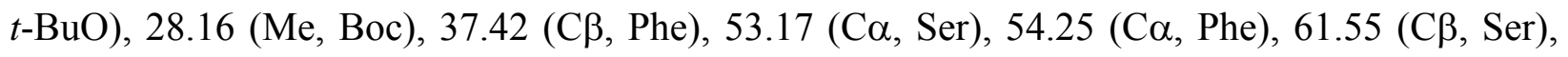


$74.04(\mathrm{C}, t-\mathrm{BuO}), 80.21(\mathrm{C}, \mathrm{Boc}), 127.04,128.49,129.9(\mathrm{CH}, \mathrm{Ar}), 135.62(\mathrm{C}, \mathrm{Ar}), 155.51(\mathrm{C}=\mathrm{O}$, Boc), 170.78 and $174.41(\mathrm{C}=\mathrm{O})$.

$\boldsymbol{N}$ - $\boldsymbol{\alpha}$-Boc-L-alanine - $\boldsymbol{S}$-p-methoxybenzyl-L-cysteine. ${ }^{1} \mathrm{H}$ RMN $\left(\mathrm{CDCl}_{3}\right) \delta 1.35(\mathrm{~d}, 3 \mathrm{H}, J=, 7$ $\mathrm{Hz}, \mathrm{Me}) ; 1.42$ (s, 9H, Me); $2.90\left(\mathrm{dq}, J=14.0 \mathrm{~Hz}, J=5.9 \mathrm{~Hz}, J=4.9 \mathrm{~Hz}, 2 \mathrm{H}, \mathrm{CH}_{2}-\mathrm{S}\right) ; 3.67$ (s, $3 \mathrm{H}, \mathrm{OMe}$ ); 4.29 (br s, 1H, CH); 4.75 (m, 1H, CH); 5.33 (br s, 1H, NH); 6.82 (d, J=8.6 Hz, 2H, Ar); 7.10 (br s, $1 \mathrm{H}, \mathrm{NH}) ; 7.20$ (d, $J=8.6 \mathrm{~Hz}, 2 \mathrm{H}, \mathrm{Ar}$ ).

$N$ - $\alpha$-Boc-L-alanine-L-leucine. ${ }^{1} \mathrm{H}$ RMN $\left(\mathrm{CDCl}_{3}\right) \delta 0.92(\mathrm{bd}, 6 \mathrm{H}, \mathrm{Me}, \mathrm{Leu}) ; 1.35$ (d, $J=7 \mathrm{~Hz}$, $3 \mathrm{H}, \mathrm{Me}, \mathrm{Ala}) ; 1.43$ (s, 9H, Me); 1.42-1.80 (m, 3H, $\left.\mathrm{CH}_{2}, \mathrm{CH}, \mathrm{Leu}\right) ; 4.20$ (br s, 1H, CH, ); 4.60 (br $\mathrm{s}, 1 \mathrm{H}, \mathrm{CH}) ; 5.26$ (br s, $1 \mathrm{H}, \mathrm{NH}) ; 5.79$ (br s, $1 \mathrm{H}, \mathrm{COOH}) ; 6.82($ br s, $1 \mathrm{H}, \mathrm{NH}) .{ }^{13} \mathrm{C} \mathrm{NMR}\left(\mathrm{CDCl}_{3}\right)$ $\delta 17.7$ (Me, Ala), 21.6, 22.8 (Me, CọLeu), 24.66 (C $\gamma \mathrm{Leu}), 28.13$ (Me, Boc), 41.0 (Cß̣Leu), 49.9 (CọAla), 50.7 (CọLeu), 80.13 (C, Boc), 155.78 (C=O, Boc), $173.0(\mathrm{C}=\mathrm{O}), 176.0(\mathrm{COOH})$.

\section{Acknowledgments}

The authors would like to thank CONICET (Consejo Nacional de Investigaciones Científicas y Técnicas - Argentina), Universidad Nacional de Rosario (Argentina), The Royal Society of Chemistry (U.K.) and Fundación Antorchas (Argentina) for financial support.

\section{References}

1. (a) Greene, T.W.; Wuts, P.G.M. Protective Groups in Organic Synthesis; $3^{\text {rd }}$ Edn.; Wiley: New York, 1999. (b) Kocienski, P.J. Protecting Groups; Thieme: New York, 1994.

2. (a) Burgess, K., Ed. Solid-phase Organic Synthesis; Wiley-Interscience: New York, 2000.

(b) Seneci, P. Solid-phase Synthesis and Combinatorial Technologies; Wiley \& Sons: New York, 2000. (c) Zaragoza Dörwald, F. Organic Synthesis on Solid Phase; Wiley-VCH, Weinheim, 2000. (d) Czarnik, A.W., Ed. Solid-phase Organic Syntheses; WileyInterscience: New York, 2001.

3. (a) Hodge, P. Chem. Soc. Rev. 1997, 26, 417. (b) Yan, B. Comb. Chem. High Throughput Screen. 1998, 1, 215. (c) Sherrington, D.C. J. Chem. Soc., Chem. Commun. 1998, 2275. (d) Vaino, A.R.; Janda, K.D. J. Comb. Chem. 2000, 2, 579. (e) Rana, S.; White, P.; Bradley, M. J. Comb. Chem. 2001, 3, 9.

4. Oran, D.; Ellard, J.; Bradley, M. J. Comb. Chem. 2002, 4, 1.

5. Zhang, A.J.; Russell, D.H.; Zhu, J.; Burgess, K. Tetrahedron Lett. 1998, 39, 7439.

6. Beyermann, M.; Bienert, M. Tetrahedron Lett. 1992, 33, 3745.

7. Sakaitani, M.; Hori, K.; Ohfume, Y. Tetrahedron Lett. 1988, 29, 2983.

8. Bajwa, J.S. Tetrahedron Lett. 1992, 33, 2955.

9. Dzubeck, V.; Schneider, J.P. Tetrahedron Lett. 2000, 41, 9953.

10. Li, W.-R.; Jiang, J.; Joullié, M.M. Tetrahedron Lett. 1993, 34, 1413.

11. Li, W.-R.; Jiang, J.; Joullié, M.M. Synlett 1993, 5, 362. 
12. Sakaitani, M.; Ohfume, Y. Tetrahedron Lett. 1985, 26, 5543.

13. Sakaitani, M.; Ohfume, Y. J. Org. Chem. 1990, 55, 870.

14. Roos, E.C.; Bernabé, P.; Hiemstra, H.; Speckamp, W.N.; Kaptein, B.; Boesten, W.H.J. J. Org. Chem. 1995, 60, 1733.

15. For a solid-phase transformation of Fmoc group into $O$-alkyl carbamates, see: Zaragoza, F.; Stephensen, H. Tetrahedron Lett. 2000, 41, 2015.

16. For a preliminary communication, see: Furlán, R.L.E.; Mata, E.G. Tetrahedron Lett. 1998, 39, 6421.

17. Jiang, J.J.; Li, W.R.; Joullie, M.M. Synth. Commun. 1994, 24, 187.

18. Barcelo, G.; Senet, J-P.; Sennyey, G. J. Org. Chem. 1985, 50, 3951.

19. Tarbell, D.S.; Yamamoto, Y.; Pope, B.M. Proc. Natl. Acad. Sci. USA 1972, 69, 730.

20. Nagasawa, T.; Kuroiwa, K.; Narita, K.; Isowa, Y. Bull. Chem. Soc. Jpn. 1973, 46, 1269.

21. (a) Salvino, J.M.; Patel, S.; Drew, M.; Krowlikowski, Orton, E.; Vassant Kumar, N.; Caulfield, T.; Labaudiniere, R. J. Comb. Chem. 2001, 3, 177. (b) McKeown, S.C.; Watson, S.P.; Carr, R.A.E.; Marshall, P. Tetrahedron Lett. 1999, 40, 2407.

22. Atherton, E.; Sheppard, R.C. Solid Phase Peptide Synthesis IRL Press: Oxford, 1989, pp 112-117.

23. Furlán, R.L.E.; Mata, E.G.; Mascaretti, O.A. J. Chem. Soc., Perkin Trans. 1 1998, 355.

24. Furlán, R.L.E.; Mata, E.G.; Mascaretti, O.A. Tetrahedron 1998, 54, 13023.

25. Steward, J.M.; Young, J.G. Solid Phase Peptide Synthesis, $2^{\text {nd }}$ Edn.; Pierce Chemical Company: Rockford, Illinois, 1984, p146. 